\title{
P043
}

\section{COLOUR CONTRIBUTION IN SPONTANEOUS IDENTIFICATION OF COPPER MATERIALS}

\author{
Hiroki Ishiyama et al.
}

DOI 10.25039/x47.2020.PO43

\section{Paper accepted for the $5^{\text {th }}$ CIE Symposium on Colour and Visual Appearance}

The paper was selected by the International Scientific Committee (ISC) for presentation at the 5th CIE Symposium on Colour and Visual Appearance, Hong Kong, CN, April 21-22, 2020, which, due to the corona pandemic, could not take place. The paper has not been peer-reviewed by CIE.

\section{(C) CIE 2020}

All rights reserved. Unless otherwise specified, no part of this publication may be reproduced or utilized in any form or by any means, electronic or mechanical, including photocopying and microfilm, without permission in writing from CIE Central Bureau at the address below. Any mention of organizations or products does not imply endorsement by the CIE.

This paper is made available open access for individual use. However, in all other cases all rights are reserved unless explicit permission is sought from and given by the CIE.

CIE Central Bureau

Babenbergerstrasse 9

A-1010 Vienna

Austria

Tel.: +4317143187

e-mail: ciecb@cie.co.at

www.cie.co.at 
$\mathrm{PO} 43$

\title{
COLOUR CONTRIBUTION IN SPONTANEOUS IDENTIFICATION OF COPPER MATERIALS
}

\author{
Ishiyama, H. ${ }^{1}$, Tanaka, M. ${ }^{2}$, Horiuchi, T. ${ }^{1}$ \\ ${ }^{1}$ Graduate School of Science and Engineering, Chiba University, JAPAN, \\ 2 College of Liberal Arts and Sciences, Chiba University, JAPAN \\ horiuchi@faculty.chiba-u.jp
}

\begin{abstract}
In this study, we conduct a psychophysical experiment based on the hypothesis that colour information affects the spontaneous identification of copper. We created six types of copper metal balls as test images using computer graphics. The surrounding colour scene was reflected on the balls of the test images. In each test image, we generated test stimuli in which the colour gamut was expanded in ten steps, such that a part of the reflected colour was deviated from the colour gamut of the copper material. In our experiment, we checked the accuracy and speed of copper material identification using rapid presentations. Experiments have shown that colour distribution in an object contributes to the spontaneous perception of metals by humans. Participants identified copper materials in only a few hundred milliseconds, and the colour gamut and speed of recognition were scene-dependent. In addition, it was found that the location where the colour gamut was expanded affected spontaneous perception.
\end{abstract}

Keywords: Material perception, Spontaneous identification, Metallic object, Visual experiment

\section{Introduction}

Metallic materials are common in our daily life. Copper has the same chromaticity as orange and reddish brown, but it can be identified as a metallic colour using higher-order physical information associated with the gloss phenomena, such as spatial light reflection. However, as we can instantly perceive that a material made of copper is metallic, it is natural to consider that complex physical information is not processed in early vision.

Conventional studies reported that humans could quickly identify materials. Motoyoshi et al. focused on the perception of surface gloss [1]. They hypothesized that simple image statistics contributed to the identification of surface gloss and showed that the skewness of the luminance histogram and sub-band filter outputs correlated to surface gloss and inversely correlated to surface albedo. They also found evidence that human observers use skewness, or a similar measure of histogram asymmetry, to judge the characteristics of surfaces. Sharan et al. investigated the speed of material categorization [2]. They displayed images classified into nine categorizes based on the material with short presentation times of 40,80 , and $120 \mathrm{~ms}$, and found that the categories could be identified quickly, requiring only $100 \mathrm{~ms}$ more than simple baseline tasks. However, they did not investigate metal objects or discuss metal perception. Based on both the results, it is expected that metallic objects can be identified using simple image features.

In this study, we conducted a psychophysical experiment based on the hypothesis that colour information affects the spontaneous identification of copper materials. Reference [1] discussed only luminance information for the perception of surface gloss. This is the first study to investigate colour information. In Ref. [3], a preliminary study was conducted. In this paper, we conduct analysis by increasing the number of test images.

\section{Analysis of copper material}

In Ref. [3], we measured the reflected colour of a copper object to analyze the properties of the colour generated by metal objects. The X-Rite ColorChecker under the illuminant at a temperature of $6000 \mathrm{~K}$ was reflected on a real copper board, and spectral power distributions 
of the original colour patch and reflected components were measured using a spectroradiometer (Konica Minolta CS-2000). Subsequently, we derived a spectral reflectance of the copper board from the data. To simulate changes in the colour gamut from the original object to the reflected colour, we used the spectral reflectance database [4], which included 24 colours of the Macbeth colour checker and 1269 colours of the Munsell colour chart. A partial dataset of the database was used as the surface spectral reflectance of the original object. Using the derived spectral reflectance of the copper board, we calculated the spectral power distribution of the reflected colour component.

Figure 1 shows the changes in the colour gamut from the original colour to the reflected colour. The green points represent the chromaticity of the original object, and the orange points represent the chromaticity of the reflected component on the copper board. The coloured area represents the colour gamut. The arrows extending from the chromaticity points show the changes in chromaticity. The colour gamut of the reflected colour generally shrank compared to that of the original object, and each metal type had a unique colour gamut.

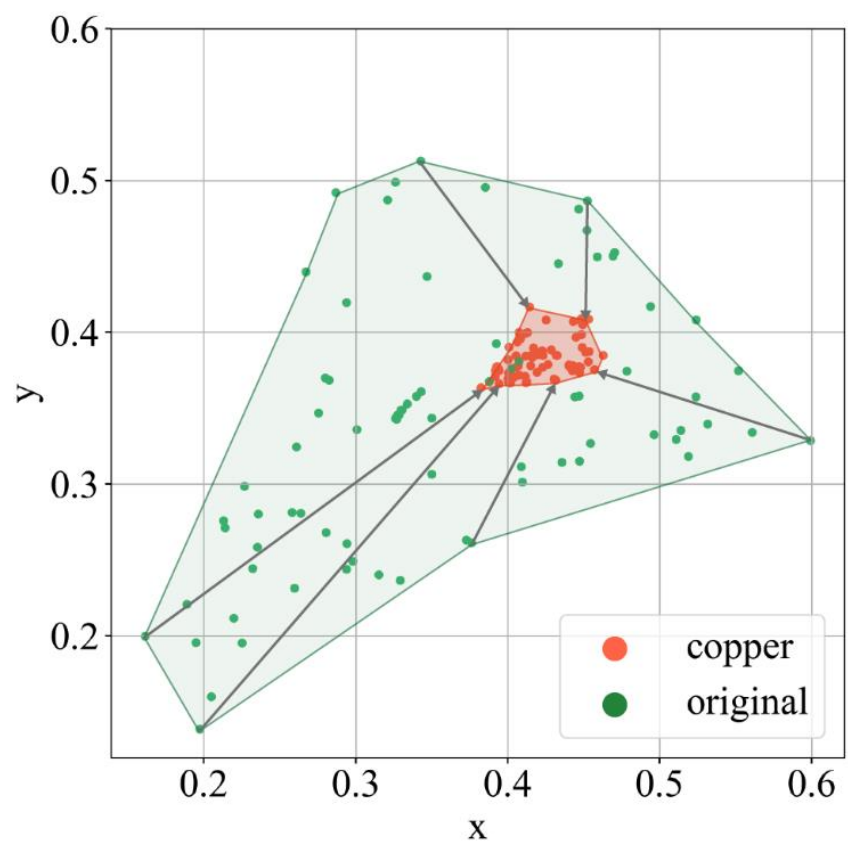

Figure 1 - Changes in the colour gamut

\section{Experiment}

\subsection{Test stimuli}

To verify whether colour information has an effect on the identification of metals, we focused on the colour gamut of the reflected colour component from a copper object. As the test stimulus, we considered using either a real metal or computer graphics (CGs). Subsequently, in this study, we decided to create a copper material image using CGs, which could freely set the colour.

The image was created using a 3D software (MAXON Computer, Cinema4D). The reflection property of a spherical surface was provided by the Trowbridge-Reitz model (GGX). Only the specular reflection was set with parameters of a metal sphere. The copper material in the layer Fresnel preset was applied. Six types of high dynamic range images in Ref. [5] were used as images of environment scenes. Therefore, six types of original scenes were generated. As these original scenes exceeded the colour gamut of the copper board from the previous section, the colour gamut was normalized such that $98 \%$ of all pixels would exist within the colour gamut. Figure 2 shows the normalized images. Further, in each spherical image, we generated 10 samples that extended the colour gamut of a specific object reflected in the image by extending the distance from the white point to the chromaticity point of each pixel at equal intervals. Scenes 1-4 have changed the colour of some of the objects, while scenes 5 and 6 have changed the colour of the entire scene. The intervals were the ratio to the distance measured subjectively 
for each normalized image, as follows Table 1. In other words, when the colour gamut of the normalized spherical image was 1 , the ratio of the maximum colour gamut for each scene was determined as follows: 6.4, 37.0, 5.5, 6.4, 4.6 and 4.6 for scenes $1,2,3,4,5$ and 6 , respectively. The ratio of scene 2 was high because the allowable range of the colour gamut of the sky region was large in the preparation experiment. Figure 4 shows a partial sample for scene 2.

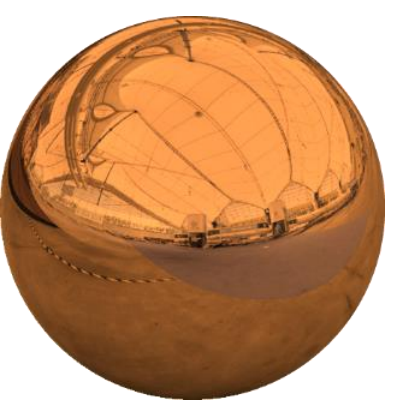

(a) Scene1

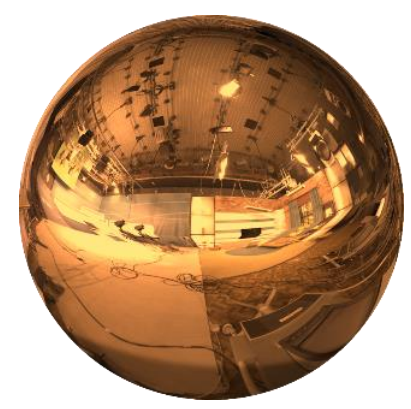

(d) Scene4

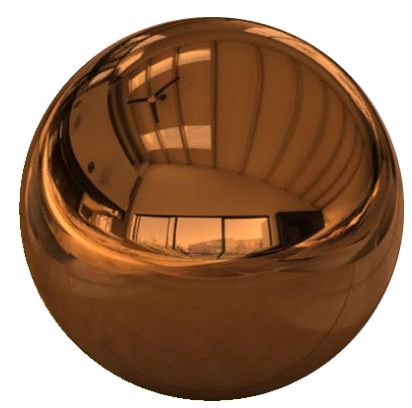

(b) Scene2

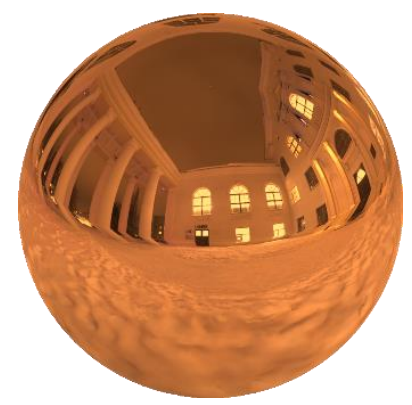

(e) Scene5

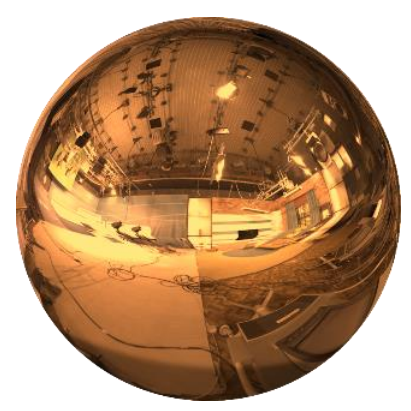

(c) Scene3

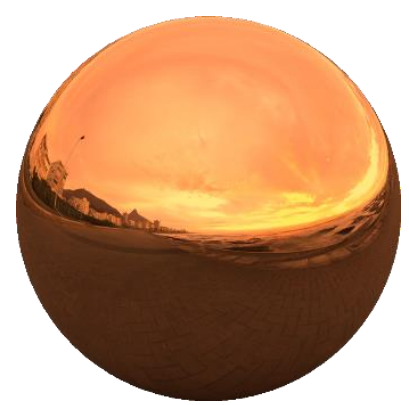

(f) Scene6

Figure 2 - Six types of normalized spherical images

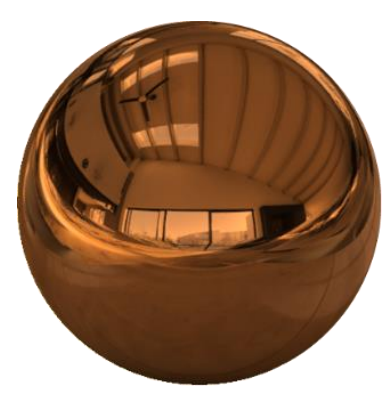

(a) Ratio = 1

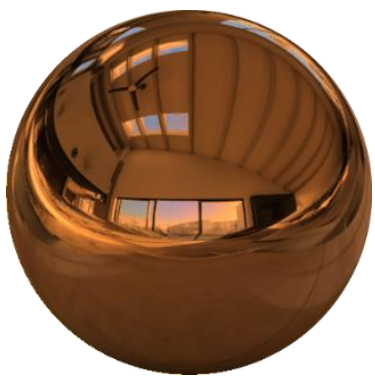

(b) Ratio $=13$

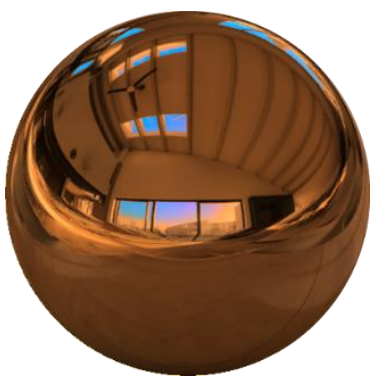

(c) Ratio $=\mathbf{2 5}$

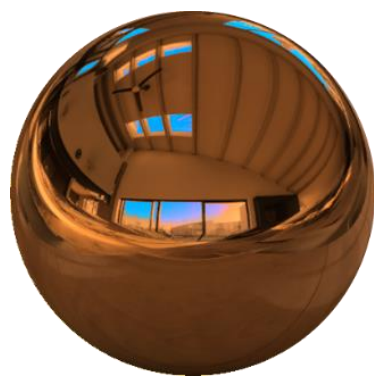

(d) Ratio $=37$

Figure 3 - A partial sample for Scene2

Table 1 - Colour gamut expansion ratio

\begin{tabular}{|l|l|l|l|l|l|l|}
\hline & Scene1 & Scene2 & Scene3 & Scene4 & Scene5 & Scene6 \\
\hline Min-Max & $1.0-6.4$ & $1.0-37.0$ & $1.0-5.5$ & $1.0-6.4$ & $1.0-4.6$ & $1.0-4.6$ \\
\hline
\end{tabular}




\begin{tabular}{|l|l|l|l|l|l|l|}
\hline Step & 0.6 & 4.0 & 0.5 & 0.6 & 0.4 & 0.4 \\
\hline
\end{tabular}

\subsection{Procedure}

The experiment followed the procedure by Sharan et al. [1], but the presentation times were 40, 80 , and $120 \mathrm{~ms}$. Their experiments aimed at categorizing materials into a single stimulus image. This study aimed at the perception of metals using chromaticity change, and the presentation time was too short to perceive differences among the stimuli. In our experiment, an initial stimulus was presented for $100,200,300,400$, or 500 ms. The initial stimulus was followed by six perceptual masks appearing for $33 \mathrm{~ms}$ (Fig. 4). We created the masks using the PortillaSimoncelli texture synthesis method [6], which matches the statistics of the mask images to the statistics of the stimulus images at multiple scales and orientations, thereby allowing for more effective masking compared to the commonly used pink noise masks. Finally, a second stimulus was presented for the same duration as the initial stimulus. In each trial, the task was to report which stimuli were perceived as a copper material. All 45 combinations of 10 samples were evaluated for each scene using two Alternative Forced Choice Task.

For all experiments, stimuli were displayed centrally on a calibrated liquid crystal display monitor (ColorEdge CG-277, EIZO) against a gray background. The vertical scanning frequency of the display was $61 \mathrm{~Hz}$, and it included the AdobeRGB colour gamut. The viewing distance was $95 \mathrm{~cm}$, and the viewing angle of the stimulus image was $12^{\circ}$, based on the experiment by Sharan et al. [1]. The experiment was performed under fluorescent lamps. Seven university students with normal colour vision participated. Figure 5 shows the experimental setup.

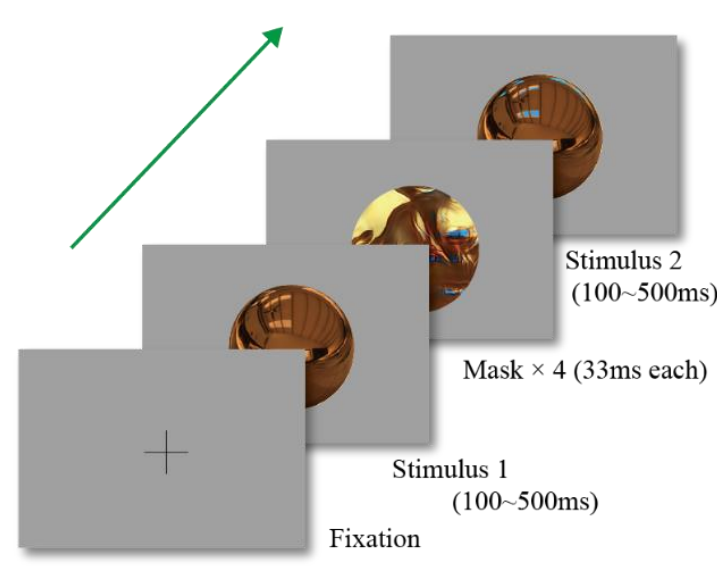

Figure 4 - Procedure of the experiment

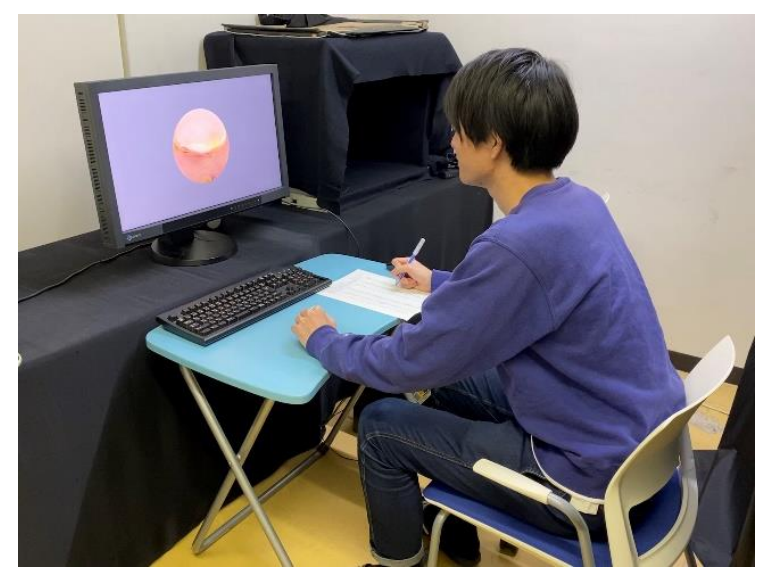

Figure 5 - Experimental environment

\section{Results}

Figures 6 and 7 shows examples of the experimental results by representing the Z-score for scene 2 and 5 , respectively. The horizontal axis indicates the magnification rate of the colour gamut of 10 samples from the normalized image, and the vertical axis indicates the $Z$-value. Smaller Z-value indicates that the stimulus is more likely to be perceived as copper. The experimental results revealed the following:

(1) Compared to the colour gamut of the reflected colours of real copper objects, stimuli could not be easily identified as copper material when they were too narrow or wide.

(2) A reproduced colour image with a colour gamut slightly wider than that of the reflection of a real copper object was easily identified as a copper material.

(3) When the presentation time was shortened, identifying the copper material was difficult. In the case of complex reflection scenes, identifying the copper material was unstable for longer presentation time as shown in Fig. 7. However, the colour gamut boundary identifiable as the copper material was stable. 
(4) The presentation time required to identify the copper material varied depending on the reflected surrounding scene.

The identification task was successfully performed when the presentation time was $300 \mathrm{~ms}$ for complex reflection scenes However, in simple reflection scenes, participants identified the copper material in less than $100 \mathrm{~ms}$, but it was unstable for the identification over $300 \mathrm{~ms}$ due to the use of features higher than colour.

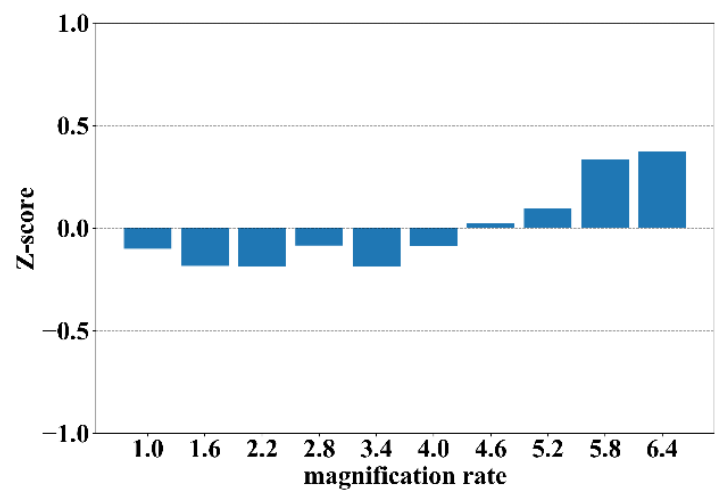

(a) $300 \mathrm{~ms}$

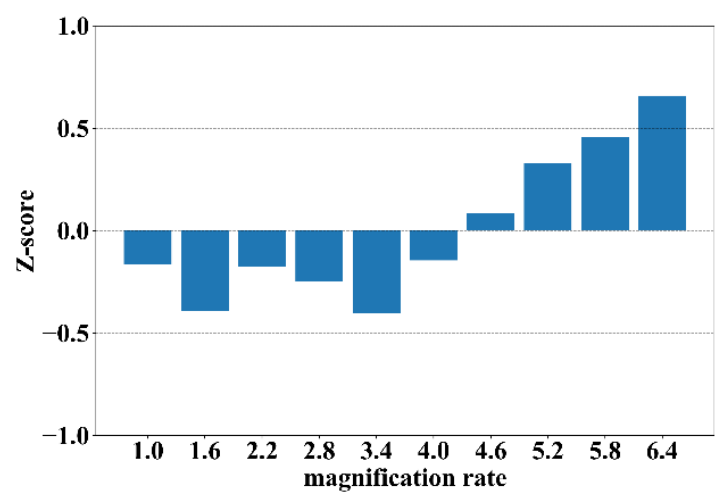

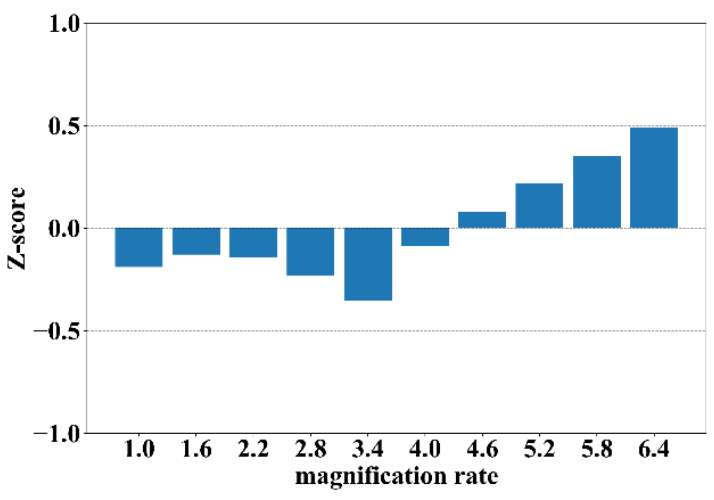

(b) $400 \mathrm{~ms}$

(c) $500 \mathrm{~ms}$

Figure 6 - Z-score for Scene 2

\section{Conclusions}

This study investigated the effect of colour information in the spontaneous perception of copper. In our experiment, we checked the accuracy and speed of copper material identification using rapid presentations. We confirmed that colour distribution in an object contributed to spontaneous perception of metals by humans. Participants identified copper materials in only a few hundred milliseconds, and the colour gamut and speed of recognition were scenedependent. In addition, it was found that the location where the colour gamut was expanded affected spontaneous perception. In the future, studies should be conducted on verification of different metals in various scenes. 


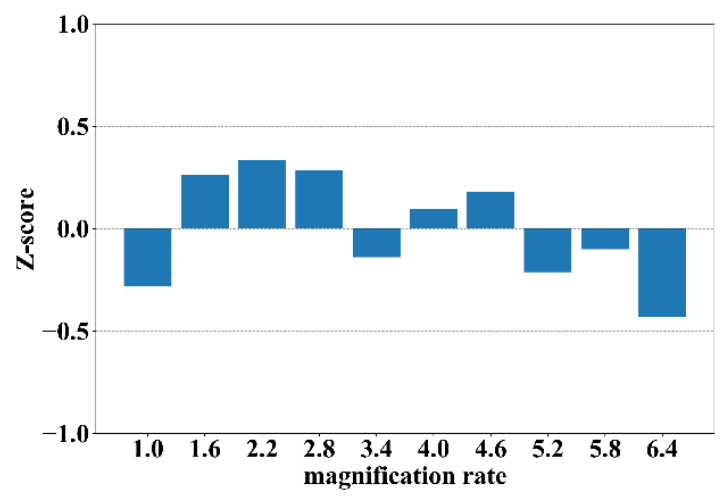

(a) $100 \mathrm{~ms}$

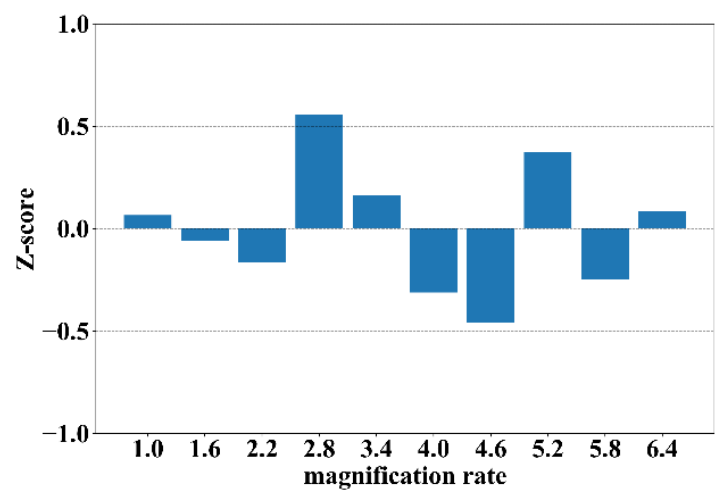

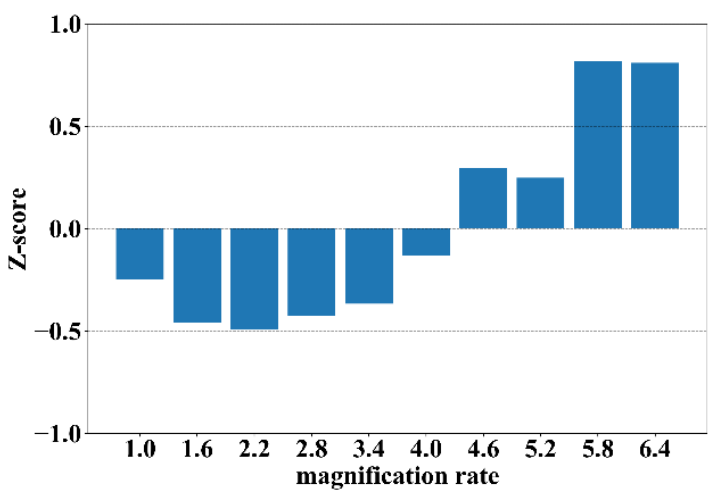

(b) $200 \mathrm{~ms}$

(c) $300 \mathrm{~ms}$

Figure 7 - Z-score for Scene 5

\section{Acknowledgment}

This work was supported by Grant-in-Aid for Scientific Research on Innovative Areas (No. 15H05926) from MEXT, Japan.

\section{References}

1. Sharan, L., Rosenholtz, R., \& Adelson, E. H. 2014. Accuracy and speed of material categorization in real-world images. Journal of Vision, 14(9), 1-24.

2. Motoyoshi, I., Nishida, S., Sharan, L., \& Adelson, E. H. 2007. Image statistics and the perception of surface qualities. Nature, 447, 206-209.

3. Ishiyama T., Tanaka M., \& Horiuchi, T. 2019. Contribution of color information in instantaneous identification of copper materials. Proc. 5th conference of the Asia Color Association ACA, 266-271.

4. Barnard, K., Martin, L., Funt, B., \& Coath, A. 2002. A Data Set for Colour Research, Color Research and Application, 27(3), 147-151.

5. sIBL Archive, http://www.hdrlabs.com/sibl/archive.html.

6. Portilla, J., \& Simoncelli, E. P. 2000. A Parametric Texture Model based on Joint Statistics of Complex Wavelet Coefficients. International Journal of Computer Vision, 40, 49-70. 